\title{
Reflexiones de futuros profesores en relación con situaciones contingentes en la sala de clases
}

\section{Reflections of Future Teachers in Relation with Contingency Situations in the Mathematics Classroom}

\author{
Roberto Araneda Benítez ${ }^{1}$ \\ Marco Uribe Santibáñez ${ }^{2}$
}

\begin{abstract}
Resumen: En este artículo se analiza la reflexión que emerge de futuros profesores de matemática cuando evalúan la gestión de situaciones contingentes en su trabajo de aula. Se triangula la información entre la estimulación del recuerdo, entrevista clínica semiestructurada y video grabación de la clase. Reportamos por medio del análisis de contenido los resultados de dos casos en los que la reflexión, permite indagar y confrontar los conocimientos visibles durante la gestión de las situaciones contingentes. Los resultados indican que los futuros profesores de matemática realizan la gestión de situaciones contingentes según el nivel de conocimiento disciplinar que poseen. Este conocimiento permite realizar una mejor gestión de clase pues le proporciona, entre otros elementos, mejores herramientas para indagar en distintos tipos de metodologías de enseñanza, uso de diversas representaciones para enseñar un determinado contenido matemático y anticiparse a errores y dificultades de los estudiantes.
\end{abstract}

Fecha de recepción: 20 de agosto de 2019. Fecha de aceptación: 14 de septiembre de 2020.

1 Escuela de Educación, Universidad de O’Higgins, Chile, roberto.araneda@uoh.cl, orcid.org/0000-00022190-3802

2 Departamento de Matemática y Física Aplicadas, Universidad Católica de la Santísima Concepción, Chile, muribe@ucsc.cl, orcid.org/0000-0002-8799-8242 
Palabras clave: Reflexión, Situaciones Contingentes, Confrontación, Cuarteto del Conocimiento.

\begin{abstract}
In this paper we analyze the reflection that emerges in future mathematics teachers when evaluating the management of contingent situations of the classroom work. The information retrieved from the stimulated recall, the semi-structured clinical interview and the video recording of the class are triangulated. We report through content analysis the results of two cases in which the reflection allows us to investigate and confront the visible measurable knowledge used for contingent situations management. The results show that future mathematics teachers manage the contingent situations according to the level of disciplinary knowledge they have. This knowledge allows them to perform better management in the class since it provides them, among others, better tools to investigate different types of teaching methods, utilization of sundry representations to teach a certain mathematical content, and anticipate mistakes and difficulties of the students
\end{abstract}

Keywords: Reflection, Contingency, Confrontation, Knowledge Quartet.

\title{
1. INTRODUCCIÓN
}

El profesor de matemática y su gestión de clase son elementos fundamentales del proceso de enseñanza. Cuando el profesor de matemática realiza su clase, debe poseer conocimientos disciplinares y didáctico disciplinarios para poder enseñar su asignatura y así ayudar a los alumnos a comprender un determinado concepto (Ball, Thames y Phelps, 2008; Torres, 2015). Según los resultados reportados el año 2015 por el Ministerio de Educación de Chile, respecto de la prueba de conocimientos INICIA, ${ }^{3} 41 \%$ de los egresados de Carreras de Pedagogía en Educación Media en Matemática de los años 2013 y 2014 posee conocimientos disciplinares insuficientes y $20 \%$ de los mismos, conocimientos didáctico-disciplinarios insuficientes, lo que hace necesario indagar en

${ }^{3}$ Prueba INICIA o Evaluación INICIA es una evaluación diagnóstica, formativa y no habilitante centrada en conocimientos disciplinares y pedagógicos donde participan egresados o titulados de carreras de Pedagogía. 
los distintos tipos de conocimientos que dispone un futuro profesor de matemática (FPM) para enfrentar sus prácticas de aula.

Durante la formación inicial de los FPM en Chile, las asignaturas Ilamadas Prácticas Progresivas (prácticas en establecimientos educacionales del sistema escolar), son las encargadas de realizar la conexión entre teoría y práctica. Es durante esta experiencia formativa, donde aparecen situaciones inesperadas o no previstas por el profesor y que requieren que se entrelacen las experiencias de aula con la teoría. Consideramos aquellas situaciones inesperadas o situaciones contingentes que, en Rowland, Huckstep y Thwaites (2005) se definen como situaciones presentes en las clases de matemática que son imprevistas e imposibles de anticipar por parte del profesor. Estas situaciones inesperadas demandan al profesor una respuesta inmediata, y no planificada previamente, por tanto, responder ante estas situaciones por parte de los FPM genera procesos de reflexión en la acción en el aula y reflexión sobre la acción fuera del aula. Rowland y Zazkis (2013) manifiestan que las situaciones contingentes en una clase de matemática ponen en evidencia dos tipos de problemas a los profesores: un problema disciplinar, claridad de la materia que está involucrada, y un problema didáctico, que facilitará el acceso y atención de los estudiantes con la solución del problema matemático. Ellos concluyen que la respuesta de un profesor a los momentos matemáticos contingentes que aparecen en la sala de clases, es importante y depende del conocimiento matemático que promueve y guía la gestión pedagógica.

En este trabajo analizamos la reflexión que emerge de futuros profesores de matemática cuando evalúan la gestión de situaciones contingentes en su trabajo de aula. Una primera pregunta que nos plantemos es: ¿Cómo los FPM consideran, gestionan y evalúan las situaciones contingentes presentes en sus clases? Para esto, se indaga en los distintos tipos de conocimiento que disponen y exteriorizan los FPM durante la gestión de situaciones contingentes. Estos tipos de conocimientos están descritos en el Mathematical Knowledge for Teaching (MKT; Ball et al, 2008) y que Turner (2012) relaciona a los momentos contingentes. La segunda pregunta es ¿Cómo la reflexión de los FPM sobre la gestión de situaciones contingentes permite reconstruir o fortalecer su práctica de aula? Para esto se confronta al FPM con su gestión de aula en una entrevista semiestructurada de modo que le permita reconstruir su trabajo de aula, para que, en futuras situaciones, posea mayor experiencia y flexibilidad ante la enseñanza de la matemática. Lo anterior obliga a relacionar tanto los conocimientos disciplinares y didáctico disciplinarios manifestados durante la gestión de situaciones contingentes. 
Un aspecto de las situaciones inesperadas tiene relación con la preparación, planificación y organización de situaciones contingentes que emergen en las salas de clases, como lo indica Hurst (2017), pues un nivel más profundo de conocimiento le permite al profesor responder de mejor manera ante momentos contingentes. La gestión de los momentos imprevistos durante el transcurso de la clase permite al profesor poner en juego distintos tipos de conocimiento disciplinar y didáctico-disciplinarios que promueven y guían su gestión pedagógica. Además, las situaciones contingentes son más difíciles de abordar en profesores recién egresados que en profesores con mayor experiencia, pues estos últimos pueden responder de mejor manera a lo no planificado (Rowland, Turner y Thawaites, 2014).

\section{MARCO DE REFERENCIA}

\section{LAS SITUACIONES DE CONTINGENCIA}

En general, el proceso de reflexión para profesores comienza cuando la experiencia de clase se torna difícil, cuando surge algún acontecimiento problemático que no puede ser resuelto inmediatamente, generando un estado de duda (Dewey, 1989). Los profesores experimentan un estado de incertidumbre que les hace volver atrás y analizar su experiencia durante o posterior a la clase. Schön (1992) propone que la reflexión durante la clase y posterior a la clase son mecanismos que los profesores reflexivos usan para su desarrollo continuo y para aprender desde sus clases. Brockbank y McGill (2002) manifiestan que la reflexión es una competencia que permite aprender de la práctica y conseguir mejorarla. En este sentido, Perrenoud (2004) establece que para formar profesores reflexivos es necesario que estos puedan disponer de un método para la reflexión, y de marcos conceptuales específicos de cada disciplina (Breda, Font y Lima, 2015; Estrella, Mena-Lorca y Olfos, 2018; Mason, 2002).

Smyth (1991) propone un modelo que lleva al docente a la reflexión sobre la práctica, pues los guía a utilizar sus propias capacidades para formular e implementar mejoras. Este es un modelo cíclico denominado Ciclo de Smyth, que comienza con la detección de un problema o de una práctica, y termina en un proceso de reconstrucción de la práctica, siguiendo las siguientes fases: 
Descripción (¿qué es lo que hago?, ¿por qué lo realizo?, ¿para qué lo realizo?).

Información (¿cuál es el sentido de la enseñanza que imparto? ¿Qué teorías sustentan la práctica?).

Confrontación (¿por qué lo realizo de esta manera?).

Reconstrucción (¿cómo podría cambiar o hacer algo diferente?).

Shulman (1987) propone que las acciones del profesor pueden ser planificadas, pero no así las respuestas de los estudiantes. Los profesores han de responder ante situaciones inesperadas que emergen durante las clases, cuyos acontecimientos son difíciles de anticipar. Estas situaciones o acontecimientos el Knowledge Quartet (KQ; Rowland, Thawaites y Jared, 2015) los considera en la dimensión contingencia, además otras tres dimensiones describen los tipos de conocimientos que se ponen en juego durante la enseñanza de la matemática. El foco de este trabajo es la dimensión contingencia, pues invita al profesor reflexionar a partir de las acciones realizadas en clase, sobre aquellos momentos inesperados y no planificados. Estas situaciones contingentes emergen generalmente mediante las contribuciones de los alumnos que, en Rowland et al. (2015) se denominan desencadenantes y establecen tres indicadores: responder a las ideas de los alumnos, reflexión del profesor durante la instrucción y la indisponibilidad de herramientas o recursos pedagógicos.

Lampert y Ball (1999) indican que los profesores deben estar preparados para lo no planificado e imprevisto, porque tendrán que decidir en el momento la gestión de aula adecuada (Mason y Spencer, 1999). El tratar con este tipo de situaciones, exige al profesor cambiar y/o modificar lo que ha planificado y por lo tanto obliga a realizar un acto de improvisación (Rowland y Zazkis, 2013). La calidad de las respuestas improvisadas por parte del profesor está determinada, en parte, por la fuente de conocimiento disciplinar y didáctico disciplinarios que tenga (Rowland et al., 2005).

\section{CONOCIMIENTOS EN LA DIMENSIÓN DE CONTINGENCIA}

Para el profesor de matemática, saber el contenido matemático es una condición necesaria para explicarla, pero no suficiente, ya que existen casos en los cuales el profesor cuenta con un buen dominio del contenido matemático, pero no es capaz de desarrollar un proceso adecuado de la enseñanza (Sosa, 
2011). Además, afirma que "el profesor deberá ser conocedor del contenido que pretende enseñar y también de los conocimientos didácticos que le permitan hacerlo" (p. 27-28). Ante esto, existe la necesidad que los profesores adquieran conocimiento del contenido (disciplinar) y conocimiento pedagógico del contenido (didáctico disciplinar), así como efectuar las interconexiones necesarias entre estos tipos de conocimientos, con el objetivo de conseguir utilizarlos de mejor manera para lograr que los alumnos aprendan matemática (Ball, 2000).

Ball et al. (2008) proponen el modelo MKT, donde centran sus investigaciones en los tipos de conocimientos matemáticos necesarios para la enseñanza, estudiando dichos conocimientos a partir de las clases del profesor. Ellos presentan dos grandes dominios de conocimiento: el conocimiento del contenido y el conocimiento pedagógico del contenido; cada uno de estos se subdividen en tres subdominios.

Tanto el modelo MKT como el KQ permiten analizar los conocimientos del FPM que se muestra en una acción de enseñanza. El modelo MKT complementa lo desarrollado por Shulman (1987) sobre el conocimiento del contenido y conocimiento pedagógico del contenido. El KQ clasifica las situaciones en las que el conocimiento matemático aparece en la enseñanza. En Turner (2009) se estudia la respuesta de profesores de primaria a las situaciones contingentes y cómo la reflexión de sus prácticas docentes les permite estar mejor preparados para actuar de forma contingente en relación con el contenido matemático y su enseñanza. En Turner (2012) se establece una conexión entre el MKT y el KQ, investigando sobre el desarrollo del conocimiento matemático para enseñar en profesores de primaria (niños de 5 a 11 años) y en situaciones contingentes, en las que se promovió tanto el conocimiento del contenido matemático como el conocimiento pedagógico del contenido, a través de la reflexión sobre la práctica docente. Como resultado de lo anterior Turner (2012) establece que los tipos de conocimientos del MKT que se visualizan en la dimensión contingencia son conocimiento común del contenido (CCC), conocimiento especializado del contenido (CEC), conocimiento del contenido y la enseñanza (CCEN) y conocimiento del contenido y los estudiantes (CCE). Estos tipos de conocimientos y su relación con la contingencia se representan en la figura 1. 


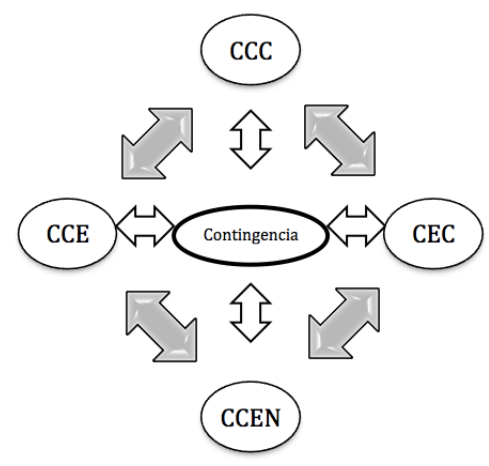

Figura 1. Relación entre el KQ y el MKT para la dimensión contingencia.

Bajo estas conexiones de conocimientos propuestas por Turner, se propone identificar los conocimientos externalizados por los FPM durante la gestión de las situaciones contingentes y establecer cómo la reflexión posterior a la clase les permite identificar aspectos mejorables de su práctica de aula futura.

\section{METODOLOGÍA}

El presente trabajo, de tipo exploratorio, se ubica en el paradigma cualitativo, siguiendo el método de estudio de caso de tipo instrumental (Stake, 2007). El trabajo se llevó a cabo con dos FPM chilenos que cursaban su último semestre de su formación profesional. En este semestre analizan y reflexionan sobre su propia práctica de manera individual y colectivamente respecto de situaciones complejas reiteradas que experimentan en el aula para proponer soluciones alternativas con aportes de su experiencia. Este nivel de reflexión es parte del Modelo de Prácticas Pedagógicas (Cisterna, 2015) para las carreras de Pedagogía de una Universidad del Sur de Chile.

Con el fin de promover el proceso de reflexión en los FPM sobre su práctica e identificar situaciones contingentes, se llevó a cabo por uno de los investigadores, la observación no participante de las clases en los establecimientos educacionales donde realizan los dos FPM su proceso de práctica profesional. Los datos de este estudio son las o notas de campo de una de las clases observadas de cada FPM, así como la videograbación de esta clase. Los registros fueron realizados en las salas de clases y los FPM siempre estuvieron acompañados del 
profesor responsable de la asignatura. Considerando criterios de relevancia de la gestión realizada por los FPM en la clase, el contenido matemático desde donde emergen las situaciones contingentes y la voluntariedad de los participantes, se reportan los resultados analizados de dos sesiones de clases correspondientes a dos FPM en contenidos matemáticos diferentes. Los contenidos matemáticos abordados por los dos FPM participantes son parte fundamental y transversal en la formación curricular de los FPM: probabilidad condicional del eje Datos y Azar en el primer caso (FPM1) e inecuaciones lineales del eje Álgebra en el segundo caso (FPM2). Ambos ejes tratados por los profesores en formación corresponden al tercer nivel de enseñanza media (niños de 16-17 años).

Junto con las notas de campo y la videograbación, se utiliza la metodología llamada estimulación del recuerdo (Carderhead, 1981) y que se obtiene por medio de una entrevista semiestructurada al FPM. Estas técnicas de recolección de información se convierten en una herramienta importante para describir la gestión de aula de las situaciones contingentes. La entrevista semiestructurada es construida con un total de trece preguntas aplicadas a cada FPM una semana posterior a la videograbación, cuyo diseño y organización fueron fundamentadas a partir de las dimensiones del Ciclo de Smyth (tabla 1).

Tabla 1. Relación entre la fase ciclo Smyth y preguntas de la entrevista

\begin{tabular}{|c|c|c|}
\hline $\begin{array}{l}\text { Fase Ciclo } \\
\text { de Smyth }\end{array}$ & Pregunta entrevista semiestructurada & Objetivos de la pregunta \\
\hline \multirow{2}{*}{ 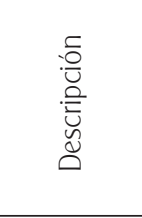 } & $\begin{array}{l}\text { ¿Recuerda la situación? Mencione qué } \\
\text { ocurre en la situación. }\end{array}$ & $\begin{array}{l}\text { Recordar la situación de aula y el máximo de } \\
\text { detalle. }\end{array}$ \\
\hline & $\begin{array}{l}\text { ¿Había previsto usted que esta situa- } \\
\text { ción apareciera, tal cual apareció en su } \\
\text { práctica de aula? }\end{array}$ & $\begin{array}{l}\text { Detectar si es una situación contingente y no } \\
\text { planificada. (Adicionalmente se realiza el se- } \\
\text { guimiento de la planificación de clases). }\end{array}$ \\
\hline \multirow{2}{*}{ 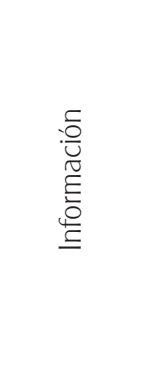 } & $\begin{array}{l}\text { Durante sus experiencias de aula en su } \\
\text { formación inicial ¿Con cuánta frecuencia } \\
\text { cree usted que aparecen estas situacio- } \\
\text { nes inesperadas en sus prácticas de aula? }\end{array}$ & $\begin{array}{l}\text { Recordar experiencias similares con situacio- } \\
\text { nes no planificadas, lo que le permite co- } \\
\text { menzar a asignar un grado de importancia a } \\
\text { dichas situaciones. }\end{array}$ \\
\hline & $\begin{array}{l}\text { Según su conocimiento y experiencia } \\
\text { de aula en su formación inicial ¿Por } \\
\text { qué surge esta situación? }\end{array}$ & $\begin{array}{l}\text { Identificar situaciones o elementos que provo- } \\
\text { can o hacen que emerjan estas situaciones } \\
\text { en la sala de clases. Podría (FPM) relacionar } \\
\text { las situaciones con las interacciones o justifi- } \\
\text { car los conocimientos previos no adquiridos. }\end{array}$ \\
\hline
\end{tabular}




\begin{tabular}{|c|c|c|}
\hline \multirow{4}{*}{ 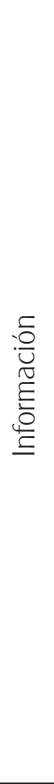 } & $\begin{array}{l}\text { ¿Qué importancia le da usted a que } \\
\text { aparezcan este tipo de situaciones en la } \\
\text { clase de matemática? ¿Por qué? }\end{array}$ & $\begin{array}{l}\text { Conocer detalles del significado personal, } \\
\text { como del significado profesional (aula) que } \\
\text { el FPM tiene de estas situaciones. Si las con- } \\
\text { sidera importante, es probable que intente } \\
\text { responder de buena manera a la situación. }\end{array}$ \\
\hline & $\begin{array}{l}\text { ¿Por qué cree usted que no se pudo } \\
\text { anticipar a la situación? }\end{array}$ & $\begin{array}{l}\text { Identificar que estas situaciones aparecen } \\
\text { únicamente en la sala de clases y no produc- } \\
\text { to de una mala planificación. }\end{array}$ \\
\hline & $\begin{array}{l}\text { ¿Por qué respondió a la situación de la } \\
\text { manera en que lo hizo? }\end{array}$ & $\begin{array}{l}\text { Justificar la gestión con alguna teoría, con- } \\
\text { ceptos matemáticos, procedimientos o activi- } \\
\text { dades realizadas previas (experiencia), donde } \\
\text { podría comenzar a reconstruir la situación, } \\
\text { mencionando aspectos para mejorar la prác- } \\
\text { tica o visualizar distintas formas de respon- } \\
\text { der, según la situación. }\end{array}$ \\
\hline & $\begin{array}{l}\text { ¿Qué tipo de conocimiento cree usted } \\
\text { necesarios al momento de responder a } \\
\text { la situación? }\end{array}$ & $\begin{array}{l}\text { Dar cuenta de aspectos formativos disciplinares, } \\
\text { didácticos-disciplinarios y/o pedagógicos, mo- } \\
\text { mento en el cuál le permite (FPM) describir y } \\
\text { justificar su gestión. }\end{array}$ \\
\hline \multirow{3}{*}{ 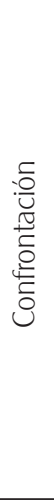 } & $\begin{array}{l}\text { ¿Cree usted que existe otra forma de } \\
\text { responder o gestionar la situación de } \\
\text { una manera diferente? Descríbala }\end{array}$ & $\begin{array}{l}\text { Identificar distintas estrategias de resolución } \\
\text { de las situaciones presentes en la práctica de } \\
\text { aula o ratificar su gestión validando las he- } \\
\text { rramientas puestas en juego. }\end{array}$ \\
\hline & $\begin{array}{l}\text { ¿Qué tipo de conocimiento pone en jue- } \\
\text { go o hace visible usted al momento de } \\
\text { responder a la situación? }\end{array}$ & $\begin{array}{l}\text { Identificar los conocimientos disciplinares, } \\
\text { didácticos-disciplinarios y/o pedagógicos } \\
\text { que hizo visibles, comparando con los men- } \\
\text { cionados anteriormente como necesarios } \\
\text { para responder ante este tipo de situaciones. }\end{array}$ \\
\hline & $\begin{array}{l}\text { ¿Cree usted que podría haber utilizado } \\
\text { un conocimiento distinto al manifesta- } \\
\text { do en el aula? ¿Por qué no lo utilizó? }\end{array}$ & $\begin{array}{l}\text { Comparar de manera explícita los distintos ti- } \\
\text { pos de conocimientos utilizado o no utilizado } \\
\text { durante la gestión y justificar cada uno de ellos. }\end{array}$ \\
\hline \multirow{2}{*}{ 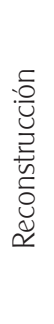 } & $\begin{array}{l}\text { Si tuviera que evaluar su respuesta o } \\
\text { gestión realizada de la situación ¿Cómo } \\
\text { la evaluaría? ¿Por qué? }\end{array}$ & $\begin{array}{l}\text { Generar con los antecedentes mencionados } \\
\text { una evaluación sobre su práctica, identificando } \\
\text { y justificando sobre aspectos a mejorar o desta- } \\
\text { car sobre lo realizado en su práctica de aula. }\end{array}$ \\
\hline & $\begin{array}{l}\text { Si se le presentan situaciones parecidas } \\
\text { a la revisada hoy que no han sido pla- } \\
\text { nificadas o esperadas ¿Cómo cree us- } \\
\text { ted que responderá? }\end{array}$ & $\begin{array}{l}\text { Reflexionar sobre la gestión realizada y men- } \\
\text { cionar aspectos a mejorar de su práctica de } \\
\text { aula. }\end{array}$ \\
\hline
\end{tabular}


Turner (2012) a partir del análisis de datos de observaciones de clases y entrevistas propone cuatro categorías para analizar los conocimientos disciplinarios y didáctico-disciplinarios visibles durante la gestión de situaciones contingentes (ver figura 1). Usando estas categorías se realiza un análisis del contenido de la información lo que permite llevar a cabo una triangulación, entendiendo esta como un proceso de estudio respecto de un mismo fenómeno, utilizando diversas estrategias para aumentar el grado de validez y hallazgos obtenidos durante la investigación (Benavides y Gómez-Restrepo, 2005). El tipo de triangulación realizado en esta investigación es entre métodos, ya que permite la verificación y comparación de la información obtenida en diferentes momentos. La triangulación en este trabajo (figura 2) está orientada principalmente a identificar los tipos de conocimientos externalizados durante la gestión de aula según los aportes realizados por Turner (2012), como los expuestos durante la entrevista. La triangulación permite validar el proceso de reflexión para complementar las dimensiones de confrontación y reconstrucción (Ciclo de Smyth) de la gestión de las situaciones contingentes.

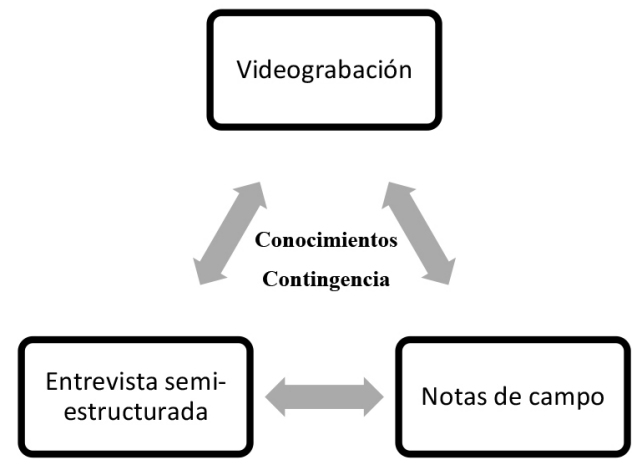

Figura 2. Triangulación entre métodos realizada en la investigación.

\section{ANÁLISIS DE LOS DATOS Y RESULTADOS}

En una primera etapa se describe el contexto general donde aparece la situación contingente, su transcripción, el desencadenante de la situación y se describe la posterior respuesta y gestión del FPM. En la segunda etapa se describen los conocimientos externalizados por el FPM durante la gestión de la situación 
contingente (Turner, 2012). En esta etapa se categorizan los datos obtenidos, en base a las características referidas en la gestión y entrevista, posteriormente se relaciona la transcripción con los conocimientos puestos en juego durante la situación contingente (ver figura 1). Finalmente, en la tercera etapa se analiza la confrontación y reconstrucción de la gestión para abordar futuras situaciones contingentes.

\section{Caso 1: Futuro Profesor de Matemática, FPM1}

En este primer caso se considera al FPM1 en el contexto de una clase de probabilidad condicional. Este contenido es parte del curriculum nacional chileno en el eje Datos y Azar y en estos últimos años ha adquirido una mayor importancia como lo muestra la Prueba de Selección Universitaria (PSU), donde 28\% de sus preguntas están orientadas al eje Datos y Azar (DEMRE, 2016). En la malla curricular de la formación de los FPM, se contemplan dos asignaturas respecto a los conocimientos del contenido (Azar y Probabilidades - Probabilidad y Estadística Inferencial) y una asignatura respecto del conocimiento pedagógico del contenido (Didáctica de la Probabilidad y Estadística). Batanero et. al. (2013) muestran que los profesores de matemática en formación tienen un bajo nivel de conocimiento de la probabilidad y su enseñanza. El manejo sobre el conocimiento del contenido por parte de los profesores afecta sus decisiones curriculares, la forma en como enseñan los conceptos y sus concepciones sobre la forma en que los estudiantes aprenden dichos conceptos (Zazkis y Leikin, 2008).

\section{Descripción general de la clase, situación de contingencia y desencadenante}

La situación contingente se genera en un tercer nivel de enseñanza media (16 a 17 años). El objetivo planteado para la clase es "comprender la probabilidad condicional y utilizar el diagrama de árbol para calcular probabilidades". La clase comienza por parte del FPM1 recordando conceptos previos y algunas fórmulas clásicas (probabilidad clásica, regla de Laplace, entre otras). Luego se introduce el concepto de probabilidad condicional a partir de la imagen proyectada en ppt de la figura 3: 


\section{PROBABILIDAD CONDICIONAL。 \\ - Sean A y B dos sucesos de un mismo espacio muestral. \\ - Se llama probabilidad de un suceso B \\ condicionado a A. \\ $\odot P(B / A)$ : SE LEE probabilidad de un suceso $B$ \\ una vez ocurrido $A$.}

Figura 3. Definición presentada por FPM1.

En el tercer punto de la figura 3, el FPM1 resalta y explica a los alumnos en forma oral la siguiente manera de resolver la probabilidad condicional:

$P(B / A)$, donde suceso B es la probabilidad a calcular y suceso A es la condición.

A partir de la imagen presentada, propone dos ejemplos proyectados en la pizarra a los alumnos. El primer ejemplo es "calcular la probabilidad de obtener un 6 al tirar un dado, sabiendo que ha salido par". El desarrollo y la respuesta dada por el FPM1 se muestran en la figura 4:

$$
P(6 / \text { par })=\frac{\frac{1}{6}}{\frac{3}{6}}=\frac{1}{3}
$$

Figura 4. Desarrollo y respuesta dada por FPM1.

Tras la resolución del problema, el FPM1 menciona:

Como quedaría ese ejercicio así... porque me están preguntando calcular la probabilidad de obtener un 6, 1 de 6 y eso va a estar dividido por mi condición A qué me dice que ha salido par y eso que significa que, si sale par, puede ser 2, 4 o 6, en este caso me preguntan por el 6 ... acá ustedes saben multiplicamos $1 / 6$ por $6 / 3$ por eso que me queda $1 / 3 . .$. la probabilidad es $0,3333 .$.

En este primer ejemplo se puede observar que la probabilidad condicional es resuelta por el FPM1 a través de la división de sucesos (suceso B y suceso A). Posteriormente proyecta un segundo ejemplo (figura 5) para mostrar la diferencia entre la probabilidad clásica y la probabilidad condicional: 


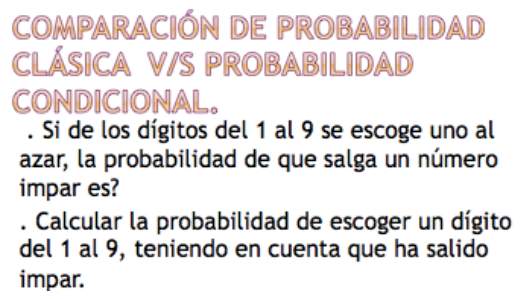

Figura 5. Segundo ejemplo: Diferencia probabilidad condicional y probabilidad clásica.

Este problema es desarrollado por el FPM1 de igual manera que el ejemplo anterior. El resultado se muestra en la figura 6.

\section{DESARROLLO \\ ๑ $\mathrm{P}$ (impar): $5 / 9$ \\ $\odot \mathrm{P}$ (dígito/par): $\frac{1 / 9}{5 / 9}=1 / 5$}

Figura 6. Resolución del FPM1 del segundo ejemplo.

Durante el resultado presentado a los alumnos el FPM1 menciona:

Esto nos quedaría así [proyecta la Figura 6], porque nos están preguntado 1 de 9 partido por la probabilidad de impar, 1 de 9 todo eso partido en 5 de 9, por lo tanto, esto quedaría 1 de 9 por 9 de 5 simplificamos y me quedaría probabilidad 1/5.

Como primer análisis de los problemas y desarrollos propuestos por el FPM1, se visualiza que la forma de resolver la probabilidad condicional es a través de la división de los sucesos ya que la fórmula es presentada como $P(B / A)$, sin mencionar lo que correctamente es: $P(B / A)=\frac{P(A \cap B)}{P(A)}$

El primer ejemplo desarrollado y resuelto, lleva al FPM1 a la respuesta correcta a pesar de que la forma de resolver no lo es, ya que coincidentemente para ese ejemplo y, por tanto, en este caso

$$
P(B / A)=\frac{P(A \cap B)}{P(A)}=\frac{P(B)}{P(A)}
$$

sin embargo, esta conclusión no es válida para el segundo ejemplo.

Finalmente, el FPM1 propone un tercer ejemplo (figura 7): 


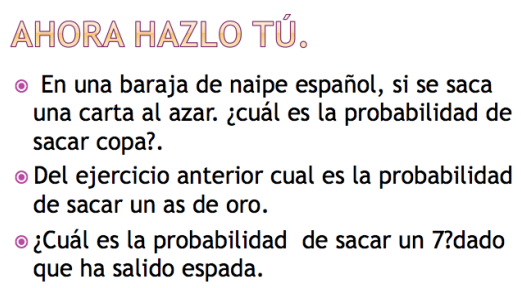

Figura 7. Tercer ejemplo propuesto por FPM1.

A partir de este problema planteado por el FPM1 y la respuesta dada por los alumnos a la pregunta: ¿Cuál es la probabilidad de sacar un 7 dado que ha salido espada?, surge la situación inesperada para el FPM1 que se transcribe a continuación:

FPM1: $\quad$ Ahora se presenta un ejercicio para ustedes. Ustedes saben que el naipe español consta de 40 cartas, a diferencia del naipe inglés, entonces ¿Cuál es la probabilidad de sacar una copa de un naipe español?... Sabemos que... [Los alumnos responden antes que el FPM1 termine de leer el problema]

Alumnos: 1 es a 4,10 es a 40...

FPM1: $\quad 10$ es a 40, 1 es a 4, muy bien... En el ejercicio 2, hacen otra pregunta. Del ejercicio anterior ¿cuál es la probabilidad de sacar un As de oro, un uno de Oro?

A1: $\quad 1$ es a 40

FPM1: $\quad 1$ es a 40 muy bien, porque hay uno solo... Ahora este último ejercicio es condicional ¿Cuál es la probabilidad de sacar un 7, dado que ha salido espada?

A1: $\quad 1$ es a $10 \ldots$

A2: $\quad$ iiiNo!!! 0,1

FPM1: $\quad$ A ver quién lo quiere hacer en el pizarrón... [Pasa el alumno A3 a resolver el problema al pizarrón, escribe:

$$
\frac{\frac{1}{40}}{\frac{10}{40}}
$$

Luego mirando hacia sus compañeros pregunta: "¿Algo así era, o no?" Finalmente, por sugerencia de los compañeros borra la primera respuesta dada y corrige en:

$$
\frac{\frac{1}{4}}{\frac{1}{10}}
$$

Entrega el plumón al FPM1 y se sienta. El FPM1 se dirige a la parte de la pizarra donde el alumno escribió la resolución para explicar su solución a los alumnos. 
FPM1: $\quad$ Aquí miren, definamos los dos sucesos que tengo [El alumno A3 interrumpe al FPM1 recordando la pregunta planteada anteriormente].

A3: ¿Cuál es la probabilidad de sacar un siete sabiendo que es espada?

A4: $\quad$ Hay diez espadas entonces abajo...

FPM1: $\quad$ En primer lugar, nos están pidiendo el suceso B, ¿Cuál es la probabilidad de sacar un 7? Si se dan cuenta en el naipe, tenemos el siete de espadas, siete de oro, siete de bastos y siete de copas... ¿Cierto?

Alumnos: Sí sí, ok... [Un alumno levanta su mano, y solicita pasar a la pizarra a resolver].

FPM1:
Sk, pase a hacerlo... [El alumno A4 pasa a la pizarra y escribe $\frac{\frac{1}{40}}{\frac{1}{10}}=\frac{10}{40}=\frac{1}{4}$, luego se

FPM1: $\quad$ Acá está entonces, un cuarto... [Mira la resolución del alumno y compara con lo planificado y resuelto en su cuaderno]... Yo lo hice así... Me preguntan ¿Cuál es la probabilidad de sacar un 7? Si yo saco un 7, mi probabilidad es cuatro...entonces si me dicen ¿cuál es la probabilidad de sacar un 7?, esa probabilidad es cuatro de cuarenta, porque de las cuarenta cartas puede haber cuatro sietes y después nos dan esta condición; dado que ha salido espada, entonces yo tengo que espadas son diez de cuarenta. ¿Por qué? Porque puede haber diez cartas de espada de las cuarenta cartas en total, entonces al desarrollar esto me quedaría, cuatro de cuarenta por cuarenta de diez... [el FPM1 anota en la pizarra $\frac{\frac{4}{40}}{\frac{10}{40}}$...y eso sería cuatro de diez.

Esa sería mi probabilidad, si simplifico me quedaría dos quintos..., pero bien ya están como entendiendo, es que esto me cuesta un poco [agrega para concluir el FPM1]

El desencadenante de la situación contingente es la respuesta incorrecta de dos alumnos a la actividad propuesta por el FPM1 en el ejemplo 3. La actividad es resolver un problema utilizando la probabilidad condicional. Los alumnos pasan a la pizarra para resolver el problema. Un primer alumno escribe:

A3: $\quad$ [Primero escribe $\frac{\frac{1}{40}}{\frac{10}{40}}$ y borra por sugerencia de compañeros y luego escribe $\frac{\frac{1}{4}}{\frac{1}{10}}$ ]

Luego de la respuesta del alumno, interviene el FPM1, donde recuerda el concepto probabilístico de sucesos asociados al problema planteado. Luego de la intervención pasa a la pizarra otro alumno el cuál escribe:

A4: $\quad$ [Escribe en la pizarra $\frac{\frac{1}{40}}{\frac{1}{10}}=\frac{10}{40}=\frac{1}{4}$ luego se sienta] 
Como se puede observar, el alumno A3 entrega inicialmente la respuesta correcta la que no es percibida por el FPM1, posteriormente el alumno A3 borra esta respuesta correcta y por sugerencia de sus compañeros escribe finalmente una respuesta incorrecta. Luego el alumno A4 entrega una respuesta parcialmente correcta, pero que en lo global es errado. El FPM1 de acuerdo con lo planificado esperaba como respuesta a esta actividad la siguiente resolución:

$\mathrm{P}(\mathrm{B} / \mathrm{A})=\frac{\frac{4}{40}}{\frac{10}{40}}$ esto sería cuatro de diez simplificando $\frac{2}{5}$,

esta resolución también está errada ya que la desarrolla a partir de la forma:

$$
P(B / A)=\frac{P(B)}{P(A)}
$$

\section{Análisis de la gestión del FPM1}

La primera respuesta dada por el futuro profesor, luego de la intervención del alumno A3, es explicar cada uno de los sucesos y la segunda respuesta, luego de la intervención del alumno A4, es proponer la resolución al problema que él había desarrollado en su planificación. Es interesante destacar que el FPM1 en ningún momento de su gestión de aula valida o corrige lo realizado por los alumnos. Sin embargo, sugiere y compara la resolución realizada por él, en su planificación, con la respuesta de los alumnos, diciendo: "Yo lo hice asi".

En primera instancia, la gestión del futuro profesor es explicar cómo los alumnos deberían resolver el problema propuesto, por medio de la determinación de cada uno de los sucesos. El FPM1 menciona que en el naipe español hay cuatro sietes: siete de espadas, siete de oro, siete de bastos y siete de copas. En este caso la respuesta espontánea de los alumnos a la explicación del FPM1 es: Sí, sí ok, dando a entender la comprensión de la resolución correcta, a lo que el alumno A4, de forma voluntaria pasa a la pizarra, donde resuelve incorrectamente el problema propuesto, al igual que la respuesta final del alumno A3. Respecto de la misma situación contingente, en una segunda instancia el FPM1 centra su gestión en mostrar a los alumnos el problema desarrollado de acuerdo con su planificación de clases. 


\section{Conocimiento matemático para la enseñanza (MKT) durante la gestión y entrevista}

En esta sección, por medio del análisis de contenido realizado a las videograbaciones de clases y la grabación en audio de la entrevista, se identifican los tipos de conocimientos externalizados durante la gestión y propuestos por Turner (2012), que tienen relación entre el MKT con la dimensión contingencia. La tabla 2 muestra las categorías de tipos de conocimientos externalizados por el FPM1 durante la gestión en la práctica de aula y durante la entrevista semiestructurada, identificando afirmaciones que se relacionan con cada una de las categorías.

Tabla 2. Categorías de conocimientos en la gestión de clases y en la entrevista

\begin{tabular}{|c|c|c|}
\hline \multicolumn{3}{|c|}{ Situación contingente: probabilidad condicional } \\
\hline $\begin{array}{l}\text { Categorías de } \\
\text { conocimientos }\end{array}$ & $\begin{array}{l}\text { Interpretación de la gestión } \\
\text { de clase }\end{array}$ & Evidencias de la entrevista \\
\hline $\begin{array}{l}\text { Conocimiento } \\
\text { común del } \\
\text { contenido, CCC }\end{array}$ & $\begin{array}{l}\text { El FPM1 posee en momentos la ha- } \\
\text { bilidad para resolver la tarea pro- } \\
\text { puesta a los alumnos, ya que se } \\
\text { visualiza conocimientos básicos de: } \\
\text { probabilidades de sucesos, evalua- } \\
\text { ción de probabilidad condicional a } \\
\text { partir de su fórmula y cálculo de } \\
\text { probabilidades condicionales sim- } \\
\text { ples, pero no así los conocimientos } \\
\text { de probabilidad condicional para } \\
\text { los ejemplos } 2 \text { y } 3 \text {. El FPM1 durante } \\
\text { la clase, siempre tiene resueltos los } \\
\text { problemas planteados a los alumnos. }\end{array}$ & $\begin{array}{l}\text { [...] el tema de las probabilidades me es com- } \\
\text { plicado explicarlo, sobre todo la condicional... } \\
\text { En cierto modo, sí tenía como miedo, porque } \\
\text { era un tema que hasta yo estaba un poco } \\
\text { complicado...estuve complicado, lo estudié y } \\
\text { practiqué antes... } \\
\text { [...] un profesor debe saber probabilidad condi- } \\
\text { cional en primer lugar y conocimientos previos... } \\
\text { [...] el profesor tiene que enfrentar estas situa- } \\
\text { ciones con más seguridad en el contenido... a } \\
\text { mí me falta más seguridad obviamente para } \\
\text { poder explicar la probabilidad condicional... }\end{array}$ \\
\hline $\begin{array}{l}\text { Conocimiento } \\
\text { especializado del } \\
\text { contenido, CEC }\end{array}$ & $\begin{array}{l}\text { El FPM1 no indaga en la naturale- } \\
\text { za del error de los alumnos. En esta } \\
\text { situación, no proporciona explica- } \\
\text { ciones matemáticas precisas ni } \\
\text { adecuadas, como tampoco inter- } \\
\text { preta la validez de la respuesta in- } \\
\text { esperada de los alumnos para la } \\
\text { resolución de la tarea planteada. }\end{array}$ & $\begin{array}{l}\text { [... YYo lo hice así y si está mal, yo te muestro lo } \\
\text { que está bien... } \\
\text { [...] un profesor debe saber cómo explicar y } \\
\text { como presentar el contenido... } \\
\text { l...] uno puede saber mucha matemática o no, } \\
\text { pero poder interpretar lo que realizan los } \\
\text { alumnos, es distinto y cuesta, yo creo que eso } \\
\text { con la experiencia en las clases y con los } \\
\text { años uno sabrá cómo interpretar y lo que tie- } \\
\text { ne que enseñar, pero cuando uno está em- } \\
\text { pezando, es todo nuevo... }\end{array}$ \\
\hline
\end{tabular}


Conocimiento del El FPM1 no construye el aprendizacontenido y la en- je a partir del tipo de razonamiento señanza, CCEn. que tienen los alumnos, en este caso propone la resolución de la problemática. El FPM1 no utiliza el error de los alumnos como una instancia de aprendizaje y así transformar la situación descrita en conocimiento para los alumnos. Propone sólo una técnica de resolución a la tarea durante la clase, no otorga tiempo para incorporar los aportes de los alumnos, y es él quien resuelve los problemas en la pizarra. El FPM1 no indaga en la posibilidad que otros estudiantes estén cometiendo el mismo error.

Conocimiento del El FPM1 no identifica ni se anticipa contenido y los es- ante las dificultades y errores que tudiantes, CCE. pueden presentar los alumnos, durante el desarrollo de la probabilidad condicional, que se evidencia en la clase. El FPM1, no anticipó que la resolución de este tipo de probabilidad presentaría tantas dificultades en los alumnos, a pesar de que, durante la gestión, el FPM1 explica cómo resolver la tarea.
[...] yo lo expliqué como yo lo sabía...

[...] en el fondo los alumnos podrían haber respondido bien o no, yo hubiera dicho igual..." ah! ya entonces está bien, porque yo lo hice igual" ... No hay una fundamentación...

[...] un profesor debe formar un entorno en que se pueda debatir... Saber utilizar bien los contextos, que por ejemplo en la probabilidad lo que más se usa son las monedas, el tema de los dados... eso hay que tenerlo bien claro...

\begin{abstract}
[...] Pensaba que me iban a comprender de inmediato, pero parece que, con las cartas, no sé si se confundieron por los tipos de cartas... pensé que iban a responder bien pero no estuvo bien...

[...] Anteriormente no habían abordado al parecer ese tema [probabilidad condicional], porque era nuevo para ellos. Sí habían abordado probabilidad, inclusive, se habían olvidado un poco, ya que el profesor (responsable del curso) anteriormente les hizo un resumen de la probabilidad clásica...
\end{abstract}

\section{Etapa de confrontación y reconstrucción de la gestión}

El FPM1 reconoce otra manera de gestionar la situación contingente, realizando una comparación entre lo que respondió y cómo podría haber respondido:

[...] Pude preguntar a los demás compañeros, ¿qué les parece el ejercicio que hizo su compañero?... y no lo hice, solamente vi que estaba malo el ejercicio, entonces ¿qué paso? ya siéntense todos y yo lo comencé a desarrollar... entonces pude haber dado la posibilidad a varios compañeros para pasar a la pizarra, para ver si tienen las mismas dificultades o quizás... pueden haber varios errores, pero eso fue lo que se vio 
a simple vista porque ellos fueron los que se ofrecieron...me faltó más seguridad al comentar que estaba malo y justificar el por qué lo que realicé yo está bueno...

En la confrontación, el FPM1 menciona su poca seguridad respecto del conocimiento disciplinar para saber si matemáticamente es correcto lo que realizaba en clases (CCC), como la falta de justificación frente al error de los estudiantes (CCEn) o una mejor gestión de la situación, mostrando solamente lo que él tenía planificado, sin considerar lo que los alumnos realizan en la clase.

Al finalizar la entrevista que guía la reflexión sobre su práctica se le pregunta al FPM1: ¿cómo enfrentaría una eventual situación contingente, que puede ser similar a la revisada u otra que esté también fuera de lo planificado? Él responde:

[...] En caso de que posea el conocimiento del contenido, responderé con más seguridad, también tendría que anticiparme a las posibles preguntas que me puedan realizar los alumnos... si por ejemplo voy a enseñar un contenido, y al practicar por ejemplo o al hacer la guía o los recursos, si me doy cuenta que tengo duda en algo... o pienso que esto me lo preguntarán, ¿ipor qué no lo veo de esta forma o de otra forma?, entonces ahí podría anticiparme para responder y trabajar más en los errores... No hacer como lo hice en esta situación, ya que me faltó especificar por qué está malo, por qué esta probabilidad está mala... debería explicar más por qué estaba malo...

En la reconstrucción, el FPM1 indica la importancia de poseer el conocimiento del contenido (CCC) para esa clase ya que este le entregará mayores herramientas para anticiparse a las preguntas de los estudiantes, visualizar los posibles errores (CCE) y mejorar la gestión de estos (CCEn).

\section{Caso 2: Futuro Profesor de Matemática, FPM2}

Los contenidos de las inecuaciones lineales dentro del curriculum nacional están presentes en el nivel de cuarto año medio (entre 17 y 18 años) y corresponden al eje Álgebra, aunque este contenido ya es abordado en el nivel de tercer año medio (entre 16 y 17 años). El desarrollo de este contenido, en general, es visto de manera procedimental, siguiendo algoritmos establecidos, donde se privilegia un trabajo mayormente algebraico. Este trabajo, también adquiere relevancia en 
la formación de los futuros profesores pues durante su preparación universitaria, el contenido de inecuaciones se trabaja de manera transversal en gran parte de las asignaturas disciplinares de la malla curricular de la carrera a la que pertenece el FPM2 (álgebra, análisis, análisis discreto, algebra lineal, probabilidades y azar, entre otras).

\section{Descripción general de la clase, situación de contingencia y desencadenante}

La situación contingente se genera en el tercer nivel de enseñanza media (entre 16 y 17 años), donde el objetivo planteado por el FPM2 para la clase es "representar de manera gráfica, intervalar y como unión de conjuntos las soluciones de inecuaciones lineales". El FPM2 comienza la clase, proponiendo encontrar el conjunto solución del sistema de inecuaciones lineales dado por:

$$
x>1 ; 1+x \geq 5
$$

Este problema fue resuelto por los estudiantes a través de distintas formas de representación. Luego de encontrar la solución a cada inecuación lineal, el FPM2 escribe en la pizarra la siguiente pregunta: ¿Si uniéramos las soluciones de las dos inecuaciones cuál sería la nueva solución?

Es en la respuesta a la pregunta abierta de un alumno, cuando emerge la situación contingente, que es analizada a continuación.

FPM2: Ya ¿que respondieron acá los compañeros?

A1: $\quad$ Que empieza desde ahí no más.

FPM2: ¿Desde dónde creen ustedes que sería la solución al unir?

A1: $\quad$ Del uno al infinito, porque hay que juntar los dos.

FPM2: $\quad$ Bien, juntando los dos, sería del uno hacia el infinito... Acá cise entiende... Acá la compañera

A2: $\quad$ iSi!

FPM2: ¿Qué respondieron ustedes?

A2: $\quad$ Que hay que colocar desde donde empieza la gráfica, hasta más allá...

FPM2: Bien... 
A2: $\quad$ Desde el uno hasta el infinito positivo [Esto lo comenta A2 considerando la siguiente gráfica propuesta en la pizarra]

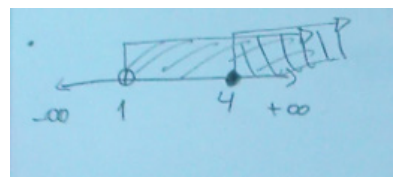

FPM2: $\quad$ Bien, otra forma sería desde donde empieza el dibujo, la gráfica hasta donde se nos indica que termina... [Indicando con la mano la gráfica de la unión de la solución de ambas inecuaciones, propuestas en la pizarra]

A3: ¿ ¿Pero si una va para allá y otra para acá? ¿Cómo quedaría? ¿infinito negativo?

FPM2: $\quad$ Acá un compañero me hacía una pregunta... Se acuerdan de que, en la guía al resolver un ejercicio, había uno que daba intersección vacía... voy a escribirlo acá

Alumnos: Sí [se escuchan a dos alumnos que responden]

A4: ¿Qué pasa en ese caso?

FPM2: $\quad$ Vamos a hacer uno... Supongamos que sea un tres y acá un cuatro, no cinco [el FPM2 realiza el dibujo en la pizarra]... Al decir intersección eso sería vacío, ¿cierto?...

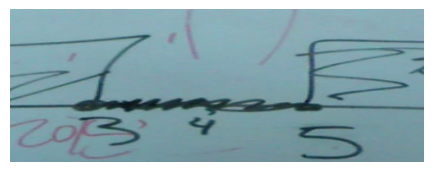

A4 y A3: Sí...

FPM2: Por qué no se juntan nunca las dos gráficas... Pero la unión es juntar todo, unir todas las soluciones, ¿Cuál sería la solución a eso? ¿Sería vacío también? O sería todo el...

A5: $\quad$ Da la vuelta completa

A5: $\quad$ Desde el cinco hasta el tres

A6: $\quad$ iNo!... si no se juntan...

A5: $\quad$ iNo! pero es que da la vuelta!

FPM2: $\quad$ A ver acá el compañero, escuchemos.

A3: $\quad$ Desde el infinito negativo hasta el tres...

FPM2: $\quad$ Hasta el tres cerrado...

A3: $\quad y$ del cinco hasta al infinito positivo... 
FPM2: $\quad$ Desde el cinco hacia el infinito positivo [El FPM2 escribe en la pizarra la respuesta del alumno]

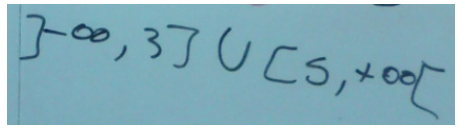

FPM2: $\quad$ Y eso sería unido ¿El cuatro estaría en la unión? [El FPM2 incluye en la gráfica de las dos soluciones de las inecuaciones lineales el cuatro]

Alumnos: iiiNo!!! [Antes de terminar la pregunta el FPM2, responden...]

FPM2: No

A7: $\quad$ Pero sería más fácil decir todos menos el cuatro.

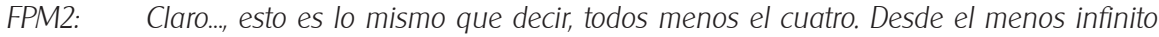
hasta el tres... Y del cinco hacia el infinito positivo... ¿Entonces se entiende? En la unión esto no representa vacío...

A3: $\quad Y$ entre los números tampoco como el tres coma dos, tres coma... lo que está entre medio tampoco puede ser...

FPM2: $\quad$ Todo esto que está aquí entre, ¿iven ustedes que pertenece a la unión? [El FPM2 marca, el intervalo entre tres y cinco de la representación gráfica de las dos soluciones]

Alumnos: No

FPM2: $\quad$ No cierto, porque está afuera... [La profesora responsable del curso sugiere representar la solución de otra forma] Acá un compañero nombró algo de los reales, que sería aquí otra forma de representar la solución... ¿Cómo sería?

A3: $\quad$ Todos los reales menos el cuatro

FPM2: $\quad$ Todos los reales, menos el elemento cuatro [EI FPM2 escribe la respuesta del alumno A3, al lado de la solución como intervalo]

A3: $\quad$ ¿Y los que están que son decimales?

FPM2: $\quad C l a r o$, bueno en este caso, si estamos trabajando con enteros sería el cuatro solamente, para que no se confundan... [La profesora responsable del curso nuevamente comenta: "Tiene razón él porque aquí, es un intervalo abierto". El FPM2 borra una parte de la solución, dejando solamente el signo de números reales y pregunta a los alumnos] ¿Ya quién?

A4: $\quad$ Del tres coma uno al cuatro coma nueve

FPM2: Del tres coma... [Cuando el FPM2 comienza a escribir la solución tres coma uno, la profesora responsable del curso le comenta: No! del tres al cuatro]

A4: $\quad$ Del tres al cuatro

FPM2: $\quad$ Del tres al cuatro... Ahí sí 
A3: $\quad$ Al cinco... ¿y el cinco?

FPM2: $\quad$ Al cinco abierto ¿si? ?.. [El FPM2 corrige el intervalo escrito en la pizarra]. Entonces una forma es que va desde el menos infinito al tres, unido con lo que va del cinco al más infinito; o también puede ser todos los reales menos lo que está entre el tres y el cinco... ¿Se entiende?

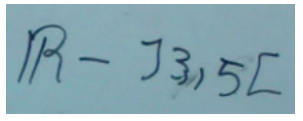

A4: $\quad$ Sí

FPM2: $\quad$ Acá las compañeras, ¿̇se entiende?

Varios alumnos: Sí

El desencadenante de la situación contingente surge a partir de una respuesta espontánea de un alumno a la pregunta planteada: ¿Cómo es la gráfica de la solución de dos inecuaciones lineales cuando se solicita unir las soluciones en vez de intersectarlas?

La situación inesperada para el FPM2 surge ante la interrogante de un alumno sobre la solución cuando la intersección está formada por sólo un punto y cuando la solución es el conjunto vacío.

\section{Análisis de la gestión del FPM2}

La respuesta del futuro profesor a la situación contingente es reconocer lo que pregunta el alumno e incorporarlo a la gestión de la clase. Inicialmente el FPM2 decide realizar su gestión de acuerdo con su planificación, pero luego modifica su trabajo incorporando el aporte del alumno A3.

La gestión realizada por FPM2 es responder a través de una actividad propuesta en la clase anterior, donde se solicitaba intersectar la solución de dos inecuaciones lineales, pero que, en este caso particular, la intersección resultaba ser el conjunto vacío. El FPM2 propone bosquejar en la pizarra la gráfica de la intersección de las dos inecuaciones lineales cuando esta es vacía, que completa con datos que surgen en el momento. El FPM2 entrega orientaciones para que los alumnos comenten, argumenten respecto de la solución en el caso de la unión y a partir de los aportes de los alumnos encontrar la respuesta a la problemática planteada. 


\section{Conocimiento matemático para la enseñanza (MKT) durante la gestión y entrevista}

En esta sección, por medio del análisis de contenido realizado a las videograbaciones de clases y la grabación en audio de la entrevista, se identifican los tipos de conocimientos externalizados durante la gestión y propuestos por Turner (2012), que tienen relación entre el MKT con la dimensión contingencia. La tabla 3 muestra las categorías de tipos de conocimientos externalizados por el FPM2 durante la gestión en la práctica de aula y durante la entrevista semiestructurada, identificando las afirmaciones dadas que se relacionan con cada una de las categorías.

Tabla 3. Categorías de conocimientos en la gestión de clases y en la entrevista

\begin{tabular}{|c|c|c|}
\hline \multicolumn{3}{|c|}{ situación contingente: inecuaciones lineales } \\
\hline $\begin{array}{l}\text { Categorías de } \\
\text { conocimientos }\end{array}$ & $\begin{array}{l}\text { Interpretación de la } \\
\text { gestión de clase }\end{array}$ & Evidencias de la entrevista \\
\hline $\begin{array}{l}\text { Conocimiento } \\
\text { común del } \\
\text { contenido, CCC }\end{array}$ & $\begin{array}{l}\text { Según la descripción del conocimiento } \\
\text { común del contenido, el FPM2 posee } \\
\text { los conocimientos que involucran la } \\
\text { tarea propuesta, no tan solo para la } \\
\text { unión sino también para la intersec- } \\
\text { ción; esto a pesar de que al final de la } \\
\text { actividad improvisada, confunde las } \\
\text { soluciones enteras con las reales, debi- } \\
\text { do a los números propuestos en el pro- } \\
\text { blema escrito en la pizarra. Según lo } \\
\text { que se evidencia en la situación, pode- } \\
\text { mos indicar que sí es posible determi- } \\
\text { nar este tipo de conocimiento durante } \\
\text { la gestión de la situación contingente. }\end{array}$ & $\begin{array}{l}\text { El FPM2 reconoce que: } \\
\text { [... El profesor de matemática debe tener el } \\
\text { dominio de lo que va a decir, de lo que uno } \\
\text { va a enseñar... El profesor de matemática } \\
\text { siempre debe tener el dominio del contenido... } \\
\text { [... me falta tener más seguridad... porque } \\
\text { sobre todo cuando me aparecen ejemplos } \\
\text { de la nada, como que me pierdo un poco... A } \\
\text { veces uno tiene pensada la respuesta co- } \\
\text { rrectamente, pero al momento de expresarla } \\
\text { verbalmente no lo hace... [El FPM2 menciona } \\
\text { que tuvo inseguridades en la respuesta den- } \\
\text { tro de la gestión de la situación]... }\end{array}$ \\
\hline $\begin{array}{l}\text { Conocimiento } \\
\text { especializado del } \\
\text { contenido, CEC }\end{array}$ & $\begin{array}{l}\text { Según la descripción del conocimiento } \\
\text { especializado del contenido, el FPM2 } \\
\text { indaga en la naturaleza de la dificul- } \\
\text { tad del alumno. El FPM2, propone a } \\
\text { los alumnos realizar una actividad } \\
\text { que ya se había desarrollado sobre la } \\
\text { intersección en la solución de dos } \\
\text { inecuaciones. Según lo que se puede } \\
\text { evidenciar en la situación, podemos } \\
\text { indicar que sí es posible evidenciar } \\
\text { este tipo de conocimiento durante la } \\
\text { gestión de la situación contingente. }\end{array}$ & $\begin{array}{l}\text { [...] Lo que uno dice en la clase, no puede } \\
\text { aparecer de la nada... uno debe interpretar } \\
\text { la respuesta de los alumnos... La seguridad } \\
\text { y como se responde... Uno puede tener una } \\
\text { muy buena respuesta, pero si la explica de } \\
\text { forma insegura el alumno va a quedar con } \\
\text { dudas y será poco claro... }\end{array}$ \\
\hline
\end{tabular}




\begin{abstract}
Conocimiento del Según la descripción del conocimiento contenido y la del contenido y la enseñanza, el FPM2 enseñanza, CCEn realiza una pausa en la clase, e incorpora el aporte del alumno para poder relacionar la intersección y unión de la solución de dos inecuaciones lineales, cuando la primera es vacío. Construye la respuesta, a partir de los aportes que se generan en la clase. También, incorpora los aprendizajes previos y actividades resueltas en clases anteriores, para resolver la interrogante generada. Propone resolver la situación a partir de una gráfica, representarla a través de intervalo y como conjunto. Por lo anterior, podemos indicar que sí es posible evidenciar este tipo de conocimiento.
\end{abstract}

Conocimiento del contenido y los estudiantes, CCE
Según la descripción del conocimiento del contenido y los estudiantes, el FPM2 se había anticipado a que los alumnos, podían tener dudas con respecto a la diferencia entre la intersección y la unión de las soluciones de dos inecuaciones, pero tenía previsto que esta surgiría cuando los alumnos resolvieran la guía de ejercicios que propondría luego en la clase. El FPM2 reconoce e incorpora el aporte del alumno a la actividad de clase. Según lo que se puede ver en la gestión de la situación, podemos indicar que sí es posible evidenciar este tipo de conocimiento.
[...] Siempre con el profesor [responsable del curso], recalcamos la diferencia entre la intersección y la unión... no hacer como clases separadas sino unirlas... relacionarla con los aprendizajes previos de los alumnos, en este caso la intersección de dos soluciones de inecuaciones, donde la intersección es vacío.

[...] en realidad esa respuesta, en algo la tenía pensada... siempre la pensé en relación con la guía, siempre la pensé así... Cuando estaba haciendo la guía, yo sabía que me iban a preguntar eso, y yo sabía que algunos iban a pensar que también era vacío y que me iban a responder vacío en la guía, de hecho, lo pensé, la respuesta la pensé planificando en relación con la intersección, siempre haciendo la relación y diferencia, por eso respondí así, siempre recalcando lo que revisamos en la guía con la diferencia que ahora es la unión.

[...] un profesor siempre tiene que anticiparse a la clase, a cualquier cosa de la clase... Uno tiene que ser consciente de que no todo lo que uno elabore va a ser tal cual, siempre van a salir detalles o cosas, que no son detalles, que van a cambiar la estructura de la clase que uno planificó. 


\section{Etapa de confrontación y reconstrucción de la gestión}

El FPM2 no reconoce otra manera de responder ante situaciones inesperadas, pues comenta:

[...] No me imagino otra manera distinta de responder...

Ante esto, el entrevistador pregunta: "¿Si te ves enfrentado a alguna situación similar, lo relacionarías de igual manera con contenidos vistos anteriormente?"

FPM2: El objetivo de la clase de la intersección y de la clase de la unión, era que al final, en la evaluación, ellos diferenciaran cuál de las dos era... Siempre con el profesor, responsable del curso, recalcamos la diferencia entre la intersección y la unión... no hacer como clases separadas sino conectarlas...

Al finalizar la entrevista que guía la reflexión sobre su práctica se le pregunta al FPM2 ¿cómo enfrentaría una eventual situación contingente, que puede ser similar a la revisada y que esté fuera de lo planificado? El FPM2 responde:

[...] Lo que nunca me ha gustado es que me pregunten y no considerarlo, no me gusta dejar sin responder una pregunta... Yo creo que la seguridad al momento de responder ante situaciones que surjan de la nada, va a depender de la experiencia que uno tenga, por ejemplo ahora mismo, quizá no tengo la experiencia suficiente, por eso al momento de responder tengo inseguridades y quizás al comienzo de mi trabajo me va a pasar lo mismo, pero a través del tiempo eso debería ir cambiando, porque uno, al ir haciendo varias clases, generalmente, las preguntas se van repitiendo y por tanto, uno ya debería anticiparse a esas cosas...

En la reconstrucción, el FPM2 indica la importancia del conocimiento común del contenido (CCC) pues este le permite anticiparse de mejor manera a las preguntas de los estudiantes (CCE) y responder con mayor seguridad en su gestión de clase (CCEn). Finalmente es conveniente indicar que el FPM2 destaca la importancia de la experiencia de aula para responder y gestionar situaciones no planificadas cómo lo indica Hurst (2017). 


\section{DISCUSIÓN Y CONCLUSIONES}

El objetivo de este trabajo es analizar la reflexión que emerge de FPM cuando evalúan la gestión de situaciones contingentes en su trabajo de aula.

Recordando la primera pregunta de investigación ¿Cómo los FPM consideran, gestionan y evalúan las situaciones contingentes presentes en sus clases?, la evidencia obtenida muestra la existencia de situaciones contingentes en ambos casos. De la entrevista semiestructurada se corrobora que el FPM1 no considera esta situación inesperada como una oportunidad de gestionar mejor los aprendizajes de los estudiantes, ajustándose solamente a lo planificado e intentando alejarse lo menos posible de esto. Por su parte, el FPM2 incluye en su gestión de aula la situación contingente como elemento de aprendizaje de los estudiantes, lo que se evidenció tanto en la gestión de aula como en el análisis reflexivo de la entrevista, debido a que menciona que esperaba la ocurrencia de esta situación, pero no planificada para ser abordada en la gestión de la clase. En ambos casos es posible afirmar lo mencionado por Rowland y Zazkis (2013) donde el tratar las situaciones contingentes invita al profesor a cambiar y/o modificar lo planificado obligando a un acto de improvisación.

Durante la entrevista, el FPM1 evidencia que su gestión puede estar condicionada por la inseguridad del conocimiento del contenido de probabilidad condicional abordado en la clase y, por tanto, mostrando debilidades en la gestión e inseguridad en el conocimiento didáctico-disciplinar como lo indica Batanero et al. (2013). El FPM2 menciona que el manejo adecuado del conocimiento del contenido de inecuaciones lineales le permitió realizar una mejor gestión de aula y por tanto este conocimiento disciplinar le ha permitido mejores oportunidades de ayudar a los estudiantes a aprender un determinado concepto, así como lo manifiesta Ball et al. (2008). Con lo anterior es posible corroborar que un nivel más profundo de conocimiento le permite al profesor responder mejor, planificar y anticipar momentos contingentes, como indica Hurst (2017).

En la segunda pregunta de investigación ¿Cómo la reflexión de los FPM sobre la gestión de situaciones contingentes permite reconstruir o fortalecer su práctica de aula?, la evidencia muestra que el FPM1 reconoce en la entrevista que para responder a situaciones contingentes el profesor debe poseer los conocimientos tanto del contenido disciplinar como didáctico disciplinarios y que estos no los externalizó debido a que manifiesta inseguridad en el conocimiento común del contenido (CCC), lo que le obstaculiza poder indagar en los otros tipos de conocimientos didáctico disciplinarios (CCE, CCEn). Por su parte, 
en la gestión realizada por el FPM2 se visualiza el conocimiento común del contenido (CCC) y conocimiento especializado del contenido (CEC) ya que la tarea matemática es resuelta de manera correcta, interpretando la naturaleza matemática de la dificultad de los alumnos. Se reconoce en el FPM2 el conocimiento del contenido y la enseñanza (CCEn) y conocimiento del contenido y los estudiantes (CCE) ya que incorpora en la gestión los aportes de los alumnos y construye de manera colaborativa una respuesta a la tarea matemática, siendo esta anticipada de manera general durante la preparación y planificación de la clase, lo que se corrobora durante la entrevista. Estos resultados se relacionan con el análisis de la primera pregunta de investigación, ya que la calidad de las respuestas improvisadas del profesor está determinada, en parte, por la fuente de conocimiento disciplinar y didáctico disciplinarios que disponga, coincidiendo con lo que afirman Rowland et al. (2005). Un aspecto importante por destacar es lo mencionado durante la entrevista por el FPM2, indicando que la experiencia del profesor facilita responder con mayor seguridad ante situaciones contingentes. Esto es afirmado por Rowland et al. (2014) donde las situaciones contingentes son más difíciles de abordar en profesores recién egresados que en profesores con mayor experiencia, pues estos últimos pueden responder de mejor manera a lo no planificado.

En los FPM el proceso confrontación y reconstrucción durante la entrevista les permitió identificar de su práctica de aula aspectos a mejorar (caso FPM1) y/o ratificar (caso FPM2) sobre los distintos tipos de conocimientos necesarios para gestionar acciones de enseñanza. Este proceso de reflexión que emerge en los FPM durante la formación puede entregarles la posibilidad de ser más flexibles ante la aparición de situaciones inesperadas que puedan ocurrir en sus prácticas de aula y por tanto modificar su planificación, incluyendo un proceso de reflexión en la acción tal como lo plantean Rowland y Zazkis (2013). Esta flexibilidad puede permitir a los FPM ser más competentes en la gestión de los momentos no planificados, fundamentando su enseñanza sobre los métodos e ideas de los alumnos que permita una mejora en los aprendizajes según indican Turner (2009, 2012), Turner y Rowland (2011) y Schön (1992). Se puede desprender de las entrevistas que los FPM visualizan en las situaciones contingentes una oportunidad para el profesor de motivar a los alumnos en la indagación, investigación o exploración a través de las respuestas de las situaciones no planificadas como menciona Rowland y Zazkis (2013).

Finalmente, es importante destacar que las situaciones contingentes son una buena herramienta para promover la reflexión durante la formación inicial de 
profesores de matemática ya que permiten confrontar y reconstruir la práctica de aula, de modo que el profesor pueda actuar de mejor manera ante la aparición de lo contingente en la sala de clases y desarrollar un enfoque de su enseñanza más cercana a la investigación de los alumnos. La estimulación del recuerdo y la entrevista semiestructurada fortalecen las herramientas del FPM que facilitan la reconstrucción de una gestión de aula futura. Esto se evidencia a partir de las declaraciones finales de la entrevista, en las que incluyen los aportes de los alumnos y consideran el actuar de manera inesperada como un factor importante dentro de la enseñanza efectiva de la matemática.

\section{REFERENCIAS}

Ball, D. L. (2000). Bridging practices: Intertwining content and pedagogy in teaching and learning to teach. Journal of Teacher Education, 51(3), 241-271. https://doi. org/10.1177/0022487100051003013

Ball, D. L., Thames, M. H. y Phelps, G. (2008). Content knowledge for teaching: What makes it special? Journal of Teacher Education, 59(5), 389-407. https://doi.org/10.1177/0022487108324554

Batanero, C., Contreras, J.M., Díaz, C. y Cañadas, G. (2013). Definición de la probabilidad y probabilidad condicional: Un estudio con futuros profesores. Revemat. 8(1), 75-91. https://doi.org/10.5007/1981-1322.2013v8n1p75

Benavides, M. O. y Gómez-Restrepo, C. (2005). Métodos en investigación cualitativa: triangulación. Revista colombiana de Psiquiatría, 34(1), 118-124.

Breda, A., Font, V. y Lima, V. M. R. (2015). A noçāo de idoneidade didática e seu uso na formaçāo de professores de matemática. Jornal Internacional de Estudos em Educaçāo Matemática, 8(2), 1-41. https://doi.org/10.17921/2176-5634.2015v8n2p\%25p

Brockbank, A. y Mcgill, I. (2002). Aprendizaje reflexivo en la educación superior. Morata.

Carderhead, J. (1981). Stimulated Recall: A method for research on teaching. British Journal of Educational Psychology, 51(2), 211-217. https://doi.org/10.1111/j.2044-8279.1981.tb02474.x

Cisterna, I. (2015). Modelos de Práctica para las Carreras de Pedagogía de la Facultad de Educación. Editorial Universidad Católica de la Santísima Concepción.

DEMRE (2016). Temario de la Prueba de Matemática. 2016.

Dewey, J. (1989). Cómo pensamos: nueva exposición de la relación entre pensamiento y proceso educativo. 1a. ed. Paidos.

Estrella, S., Mena-Lorca, A. y Olfos, R. (2018). Lesson Study in Chile: a very promising but still uncertain path. En M. Quaresma, C. Winsløw, S. Clivaz, J. da Ponte, A. Ní Shúilleabháin, 
y A. Takahashi (Eds.), Mathematics lesson study around the world: Theoretical and methodological issues, (105-122). Springer. https://doi.org/10.1007/978-3-319-75696-7

Hurst, C. (2017). Provoking contingent moments: Knowledge for 'powerful teaching' at the horizon. Educational Research, 59(1), 107-123. https://doi.org/10.1080/00131881 .2016 .1262213

Lampert, M. y Ball, D. L. (1999). Aligning teacher education with contemporary K-12 reform visions. En L. Darling-Hammond y G. Sykes (Eds.), Teaching as the learning profession: Handbook of policy and practice (33-53). Jossey-Bass.

Mason, J. (2002). Researching your own practice. The discipline of noticing. RoutledgeFalmer.

Mason, J. y Spencer, M. (1999). Beyond mere knowledge of mathematics: the importance of knowing-to act in the moment. Educational Studies in Mathematics, 38, 135-161. https://doi.org/10.1023/A:1003622804002

Perrenoud, P. (2004). Diez nuevas competencias para enseñar: Invitación al viaje. Graó.

Rowland, T., Huckstep, P. y Thawaites, A. (2005). Elementary Teachers' Mathematics Subject Knowledge: The Knowledge Quartet and the Case of Naomi. Journal of Mathematics Teacher Education, 8(3), 255-281. https://doi.org/10.1007/s10857-005-0853-5

Rowland, T., Thawaites, A. y Jared, L. (2015). Triggers of contingency in mathematics teaching. Research in Mathematics Education, 17(2), 74-91. https://doi.org/10.1080/147 94802.2015.1018931

Rowland, T., Turner, F. y Thawaites, A. (2014). Research into teacher knowledge: a stimulus for development in mathematics teacher education practice. ZDM Mathematics Education, 46(2), 317-328. https://doi.org/10.1007/s11858-013-0564-9

Rowland, T. y Zazkis, R. (2013). Contingency in the Mathematics Classroom: Opportunities Taken and Opportunities Missed. Canadian Journal of Science, Mathematics and Technology Education, 13(2), 137-153. https://doi.org/10.1080/14926156.2013.784825

Schön, D. A. (1992). Formación de profesionales reflexivos: Hacia un nuevo diseño de la enseñanza y aprendizaje en las profesiones. Paidós.

Shulman, L. (1987). Knowledge and teaching: Foundations of the new reform. Harvard Educational Review, 57(1), 1-23. https://doi.org/10.17763/haer.57.1.j463w79r56455411 Smyth, J. (1991). Una pedagogía crítica de la práctica en el aula. Revista de Educación, 294, 275-300.

Sosa, L. (2011). Conocimiento Matemático para la enseñanza en bachillerato. Un estudio de dos casos. Tesis Doctoral. Universidad de Huelva.

Stake, R.E. (2007). Investigación con Estudios de Casos. 4 ed. Morata.

Torres, M. (2015). El conocimiento del profesor de matemáticas en la práctica: enseñanza de la proporcionalidad. Tesis Doctoral. Universidad Autónoma de Barcelona. 
Turner, F. (2012). Using the Knowledge Quartet to develop mathematics content knowledge: the role of reflection on professional development. Research in Mathematics Education, 14(3), 253-271. https://doi.org/10.1080/14794802.2012.734972

Turner, F. (2009). Developing the Ability to Respond to the Unexpected. Informal Proceedings of the British Society for Research into Learning Mathematics, 29(1), 91-96.

Turner, F. y Rowland, T. (2011). The Knowledge Quartet as an organizing framework for developing and deepening teachers' mathematics knowledge. En T. Rowland y K. Ruthven (Eds), Mathematical Knowledge in Teaching (195-212). Springer.

Zazkis, R. y Leikin, R. (2008). Exemplifying definitions: a case of a square. Educational Studies in Mathematics, 69, 131-148. https://doi.org/10.1007/s10649-008-9131-7

MARCO URIBE SANTIBÁN̄EZ

Dirección: Universidad Católica de la Santísima Concepción Departamento de Matemática y Física Aplicadas Alonso de Ribera 2850, Concepción, Chile

Teléfono: $\quad+56412345688$ (Oficina) +56 952160572 (Portable) 